J. Dairy Sci. 95:4141-4152

http://dx.doi.org/10.3168/jds.2011-4550

(C) American Dairy Science Association ${ }^{\circledR}, 2012$.

\title{
Reduction in incidence of Johne's disease associated with implementation of a disease control program in Minnesota demonstration herds
}

\author{
L. A. Espejo, ${ }^{1}$ S. Godden, ${ }^{\star}$ W. L. Hartmann, $†$ and S. J. Wells ${ }^{\star}$ \\ *Department of Veterinary Population Medicine, College of Veterinary Medicine, University of Minnesota, St. Paul 55108 \\ †Minnesota Board of Animal Health, St. Paul 55155
}

\begin{abstract}
This prospective longitudinal observational study was conducted to evaluate the effect of a standardized control program on the incidence of Johne's disease in 8 dairy herds in Minnesota. Depending on recruitment year, herds were followed for between 5 and 10 yr. Program compliance was evaluated using a cohort risk assessment score by birth cohort. Fecal samples from cows in study herds were tested annually using bacterial culture to detect Mycobacterium avium ssp. paratuberculosis (MAP), and serum samples from study cows were tested using an ELISA to detect antibodies to MAP. Clinical Johne's disease was also recorded. Cohort risk assessment score decreased along birth cohorts. Depending on the follow-up period in each herd, 5 to 8 birth cohorts were followed to describe changes in time to MAP bacterial culture positivity, serum ELISA positivity, MAP heavy shedding status, and clinical Johne's disease. The analysis of time to bacterial culture positivity, serum ELISA positivity, heavy fecal shedding status, and clinical Johne's disease using a time-dependent Cox regression indicated a reduction of the instantaneous hazard ratio by birth cohorts and by cohort risk score; however, the strength of association between the cohort risk score and each of the 4 disease outcomes decreased over time. The age at which the cows first tested positive for bacterial culture, serum ELISA, and heavy fecal shedding, and the age of the cows at onset of clinical Johne's disease signs remained constant for all birth cohorts. Based on herd risk scores, overall herds complied with the recommended management practices in the program. Results were consistent with a within-herd reduction of Johne's disease transmission, and that reduction was associated with herd-level management practices implemented as part of the control program.
\end{abstract}

Received May 18, 2011.

Accepted February 10, 2012.

${ }^{1}$ Corresponding author: espe0048@umn.edu
Key words: Johne's disease, disease control program

\section{INTRODUCTION}

Paratuberculosis or Johne's disease (JD) is a chronic enteritis in ruminants caused by Mycobacterium avium ssp. paratuberculosis (MAP; Gardner et al., 2011) with a long incubation period (Hutchinson, 1996). In 2007, the US Department of Agriculture Animal and Plant Health Inspection Service (USDA-APHIS) estimated that at least $68 \%$ of US dairy farms were infected with MAP, including $95 \%$ of herds with $>500$ cows, based on testing of environmental fecal samples (USDA-APHIS, 2008). Johne's disease causes economic losses directly associated with premature culling as well as reduced milk production (Raizman et al., 2007). Moreover, JD negatively affects the welfare of cattle, because it has a slowly progressing detrimental effect on cow health, producing protein-losing diarrhea and weight loss, which can lead to death. A practical treatment of the disease is not available.

In 1999, the Voluntary Bovine Johne's Disease Control Program (VBJDCP) was implemented in the United States and was available for all cattle herds. One of the objectives of this program was to recommend implementation of specific herd management changes to reduce the risk of disease transmission (USDA-APHIS, 2010). In Minnesota, the Johne's Disease Demonstration Herd Program (JDDHP) started in 2000 and was later established at the national level by the USDA in 2003. The JDDHP followed the recommendations of the VBJDCP and focused on evaluation of the effectiveness and feasibility of management-related herd practices to control JD over a period of several years in a selected group of dairy herds, based on the best scientific information available at that time. The objectives of the JDDHP were (1) to establish management practices to minimize transmission of the disease; $(2)$ to reduce environmental contamination from MAP fecal shedders; and (3) to prevent introduction of infected cattle from other infected herds (Ferrouillet et al., 2009). 
Different strategies to control JD have been evaluated using simulation modeling to assess the effect of varying combinations of control strategies, including herd management, as well as testing and removal. Kudahl et al. (2008) showed that control strategies that included management practices focused on reduction of transmission of the disease were the most effective in reducing prevalence. Similar findings were previously reported by Groenendaal and Galligan (2003) in another simulation model of mid-size dairy farms in the United States. Those authors concluded that testing and culling test-positive cows alone does not stop disease transmission and does not reduce the prevalence of JD in the long term.

In these simulations, management practices were focused on reducing transmission between adult and younger cattle; however, a meta-analysis of 8 publications indicated that adult cattle could also become infected when they are exposed to environments with high levels of MAP contamination (Windsor and Whittington, 2010). Similarly, Mitchell et al. (2008), using simulation models, found that transmission of MAP among adult cattle in combination with vertical transmission could explain the maintenance of herds with low levels of prevalence.

Observational studies in Pennsylvania and Wisconsin have demonstrated a reduction in the apparent prevalence of JD in cattle herds under a control program (Benedictus et al., 2008; Collins et al., 2010). The study in Pennsylvania included only one herd and reported an approximately $50 \%$ decrease in the within-herd apparent prevalence of disease over an 18-yr period after implementation of management practices to reduce transmission along with testing and culling (Benedictus et al., 2008). In the Wisconsin study, an overall decrease of approximately $50 \%$ in the apparent ELISA seroprevalence was noted in 9 dairy herds after $6 \mathrm{yr}$ in the program (Collins et al., 2010). Studies with larger sample sizes have been conducted in Denmark and Australia. The Danish study included approximately 1,100 herds and reported a decrease in the seroprevalence of antibodies against MAP associated with specific JD disease management practices, such as culling repetitively serum-positive cows, used of waste milk to feed calves, and purchasing cattle from other herds (Nielsen and Toft, 2011). The Australian study included cattle from 542 herds under a disease test and control program, and showed a constant decrease in the seroprevalence of JD and clinical JD during $10 \mathrm{yr}$ of follow up (Jubb and Galvin, 2004). Even though prevalence may be useful to study changes in the frequency of the disease in the long-term, incidence is a more appropriate parameter to study changes in disease transmission, and therefore a better measurement to evaluate the effect of a disease control program.

In the Minnesota JDDHP program, 8 dairy herds were enrolled with a follow-up period that varied from 5 to $10 \mathrm{yr}$, depending on the date of herd enrollment. A partial evaluation of the Minnesota project included 6 of the 8 enrolled dairy herds for a 5-yr period from 2000 to 2005. Unlike other studies that focused on apparent prevalence, the Minnesota study demonstrated a reduction in the incidence of test-positive cattle after $5 \mathrm{yr}$ in the program and suggested a reduction in transmission of MAP because of well-implemented management practices focused on reducing transmission and testing and culling positive cattle (Ferrouillet et al., 2009). The disease control program in Minnesota has continued for an additional $5 \mathrm{yr}$, and a complete evaluation of the data was needed to evaluate the long-term effect of the control program on the incidence of JD, taking into account differences among herds.

The objectives of this study were (1) to evaluate the levels of implementation of the recommended management practices in study herds; (2) to evaluate changes in JD incidence in study herds, and whether changes in the JD incidence of disease were explained by the level of implementation of the recommended management practices through time; and (3) to evaluate if the recommended management practices delayed the onset of test positivity or clinical JD in the cows in these populations.

\section{MATERIALS AND METHODS}

\section{Herd Selection and Characteristics}

Eight dairy herds were enrolled in the JDDHP in Minnesota based on history of JD in the herds, assessed by the occurrence of clinical JD before study initiation, and owner willingness to participate in the program and cooperate with their herd veterinarian, University of Minnesota researchers, and the Minnesota Board of Animal Health. Another criterion for herd enrollment was owner motivation to control and eliminate JD in the long term, including a plan to remain in operation for at least $5 \mathrm{yr}$. All study dairy herds were members of the Minnesota DHIA. The direct costs of the JDDHP, including herd visits and testing, were funded by the Minnesota Board of Animal Health and USDAAPHIS-Veterinary Services. Herds were enrolled from 2000 to 2004 and remained on the program until 2008 or 2009. Therefore, depending on the year of enrollment and the number of years in the program, herds were followed for between 5 and $10 \mathrm{yr}$. Seven of the 8 herds used freestall housing and 1 used tiestall housing 
Table 1. Characteristics of the 8 dairy herds enrolled on the Minnesota Johne's Disease Demonstration Program at the beginning of the program (year of enrollment)

\begin{tabular}{|c|c|c|c|c|c|c|}
\hline Herd & $\begin{array}{l}\text { Housing } \\
\text { system }\end{array}$ & $\begin{array}{c}\text { Herd } \\
\text { size }\end{array}$ & $\begin{array}{l}\text { Fecal culture } \\
\text { prevalence, } \%\end{array}$ & $\begin{array}{l}\text { Serum ELISA } \\
\text { prevalence, \% }\end{array}$ & 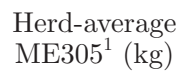 & $\begin{array}{l}\text { Follow-up } \\
\text { period }\end{array}$ \\
\hline 1 & Freestall & 269 & 14.7 & 28.5 & 11,104 & 2000-2009 \\
\hline 2 & Freestall & 42 & 38.3 & 8.3 & 9,698 & $2000-2008$ \\
\hline 4 & Freestall & 181 & 11.5 & 8.8 & 15,592 & $2000-2008$ \\
\hline 5 & Tiestall & 44 & 15.9 & 18.2 & 10,561 & $2001-2008$ \\
\hline 6 & Freestall & 246 & 15.9 & 6.5 & 11,595 & 2004-2008 \\
\hline
\end{tabular}

${ }^{1}$ Mature-equivalent 305-d milk yield.

as the primary housing system for lactating cows. The mean herd mature-equivalent 305-d (ME305) milk production was $11,240 \mathrm{~kg}$ (Table 1). Holstein was the predominant breed in all herds.

\section{Recommended Management Practices}

Herd owners and managers agreed to implement the following recommendations of the disease control program (Ferrouillet et al., 2009):

1. Clean, dry maternity area protected from manure from other adult cattle;

2. Separation of newborn calves from adult cattle as soon as possible after birth;

3. No use of pooled colostrum; instead, use colostrum from single identified, healthy, low-risk or test-negative cows;

4. Use of milk replacer or pasteurized milk to feed calves;

5. Minimal exposure of calves and heifers to the manure of mature cattle;

6. Minimal contamination of feed and water fed to young stock by manure from adults and no feeding of adult cattle refusal feeds;

7. Identification and culling of adult cattle contributing most to farm's infection load (MAP heavy fecal shedders) by testing or clinical observation in order to market them early or separate them from other cattle;

8. Acquisition of new cattle from low risk herds or testing with a fecal culture and blood for ELISA at the time of purchase;

9. Use of separate equipment for manure cleaning and feed handling.

In this study, the JD disease control program consisted of the incorporation of these recommended management practices, evaluation of their level of implementation using a risk assessment score, and evaluation of their effectiveness by measuring changes in the incidence of test positives and clinical JD.

\section{Herd Visit and Risk Assessments}

All herds were visited annually by JD-certified veterinarians, who performed the activities related to the VBJDCP described in more detail in the VBJDCP Program Standards (USDA-APHIS, 2010). Briefly, the VBJDCP Program Standards are focused on education, evaluation of the risk of transmission, and testing. Risk of JD transmission was evaluated using a risk assessment tool, which is a survey to evaluate the risk of JD transmission in 6 specific production areas: calving area, preweaned heifer calves, postweaned heifers, bred heifers, cows, and replacements and additions to the herd. Numerical scores were assigned to indicate the level of perceived risk in each of the sections of the risk assessment; therefore, the score scale differs in each production section as a reflection of changes in JD perceived susceptibility in cattle by age. The annual risk assessment scores (ARAS) for calving area, preweaned heifers calves, postweaned heifers, bred heifers, and cows varied from 0 to 80,0 to 50,0 to 30,0 to 24 , and 0 to 16 , respectively. The sum of the scores for these 5 sections represented the total ARAS for the herd and could therefore vary from 0 to 200 . The official VBJDCP program and the ARAS considered an additional section (replacements and additions) that evaluated the risk of introduction of cattle infected with JD; however, the current study did not consider these data, because the section was added to the total ARAS 3 yr after the beginning of the Minnesota JDDHP.

Although guidelines have not been established for ARAS interpretation, higher scores correspond to higher perceived risk and lower scores correspond to lower perceived risk (MNBAH, 2005; USDA-APHIS, 2010). Details of the ARAS application within the Minnesota JDDHP have been presented previously (Ferrouillet et al., 2009). As part of the follow-up plan, risk assess- 
ments were performed by trained veterinarians at the beginning of the program and again each year in approximately the same month.

\section{Sampling and Testing}

Sample collection and cattle handling procedures were approved and conducted in accordance with the University of Minnesota Institutional Animal Care and Use Committee regulations. Individual blood and fecal samples were collected from each adult cow $>24$ mo of age at least once a year in 6 herds on the same day and in approximately the same month each year. In the first $5 \mathrm{yr}$ of the program, the other 2 herds scheduled biologic sample collection based on stage of the lactation of the cows, although this was changed to annual same-day herd testing in the later years of study. Blood samples were obtained from the caudal (tail) vein using a sterile needle and a 7 - or $10-\mathrm{mL}$ evacuated blood collection tube. Blood samples were allowed to clot and serum was collected for laboratory testing. Fecal samples were obtained directly from the rectum using separate plastic sleeves, and a minimum of $10 \mathrm{~g}$ of feces was collected in sample containers. Serum and fecal samples were refrigerated $\left(4^{\circ} \mathrm{C}\right)$ and sent to the Minnesota Veterinary Diagnostic Laboratory (St. Paul) within $24 \mathrm{~h}$ after collection. Serum samples were tested using a serum ELISA (Idexx Laboratories Inc., Westbrook, ME). Fecal samples were tested using bacterial culture of feces using sedimentation for $72 \mathrm{~h}$ followed by culture on Herrold's egg yolk medium on 4 slants (Wells et al., 2002). Positive samples for serum ELISA were defined as those with a sample to positive ratio $\geq 0.25$. Similarly, positive fecal bacterial culture results were detected when the average of colony-forming units on the 4 slants was $>0$. Heavy fecal shedding cows were defined as cows with bacterial culture results $\geq 50 \mathrm{cfu} /$ slant on any of the slants for bacterial culture. Similarly, light fecal shedding cows were defined as cows with bacterial culture results $>0$ and $<50 \mathrm{cfu} /$ slant on any of the slants for bacterial culture. Positive test results were used as indication of the JD infection status of the cattle.

\section{Cow Data}

Individual cow data, including date of birth, source of the cow (purchased or home-raised), lactation number, ME305 milk production, and culling information (date and reason) were downloaded from the Minnesota DHIA (DairyComp 305, Valley Agricultural Software, Tulare, CA) at least every 6 mo for each herd for the duration of the program. Herd managers were asked to record whether a cow was culled for clinical JD (defined by chronic diarrhea and weight loss with or without a confirmatory diagnostic test result). Based on the date when the program started for each farm, birth cohorts were created. Cows born between 24 and 12 mo before the program started were defined as birth cohort -2 in the study, and cows born between 12 and 0 mo before the program started were defined as birth cohort -1 . Similarly, cows born between 0 and 12, 12 and 24, 24 and 36,36 and 48,48 and 60 , and $>60$ mo after the program started were defined as cohorts $0,1,2,3,4$, and 5 , respectively. Therefore, 8 birth cohorts $(-2,-1$, $0,1,2,3,4$, and 5 ) were created based on the date the program started in each herd. All cows that were at least $2 \mathrm{yr}$ old at time of testing and born after $60 \mathrm{mo}$ of the initiation of the program were included in cohort 5 . Cows that were $>24$ mo of age before the beginning of the program were not included in this analysis. All cattle in the different cohorts were followed for a period up to 60 mo of age. For the analysis, cattle were right-censored at $60 \mathrm{mo}$ of age to allow enough time for the latent-infected cows to become test positive, heavy fecal shedders, or clinical JD, and to include a similar number of cows in each birth cohort.

Even though the total ARAS represents an estimation of the exposure to MAP of all the cattle in a herd in a specific year, the total ARAS does not allow assessment of the level of exposure to MAP of the different birth cohorts. Therefore, a cohort risk score (CRS) was created to characterize the exposure risk to MAP of cows from birth to adulthood. A CRS was calculated for the 8 birth cohorts on each farm using the sum of ARAS from 3 consecutive years by stages of life as follows: the ARAS of the calving area, preweaned heifer calves, and postweaning heifers from the year when calves in that birth cohort were born; the ARAS of bred heifers $1 \mathrm{yr}$ after the cohort was born; and the ARAS of cows and bulls 2 yr after the cohort was born. The ARAS was not performed $1 \mathrm{yr}$ in 2 herds; therefore, the closest risk assessment score by date in the herd was used instead.

\section{Analysis}

Different statistical models were performed to (1) evaluate the levels of implementation of the recommended management practices, by evaluating changes in the CRS (evaluation of program compliance); (2) evaluate changes in MAP test-positive and clinical JD incidence and its association with the level of implementation of the recommended management practices (association of incidence and recommended management practices); and (3) evaluate if the recommended management practices delayed the onset of MAP test positivity or clinical JD in the cows in these populations (age at onset of JD). 
Evaluation of Program Compliance. To evaluate changes in the level of MAP exposure of birth cohorts, the CRS was analyzed using a repeated-measures exponential regression. The natural logarithmic transformation of the CRS was used as the dependent variable and birth cohorts as an independent variable. The working correlation structure of CRS within farm was first-order autoregressive and chosen using the Akaike's information criterion. The model was fitted using Mixed procedure of SAS (version 9.2, SAS Institute Inc., Cary, NC).

Association of JD Incidence and Recommended Management Practices. Kaplan-Meier survival curves were used to model each of the 4 primary outcomes of the study: (1) time from 24 mo of age to age at first positive result of bacterial culture of feces, (2) time from 24 mo of age to age at first heavy shedding result from bacterial culture of feces, (3) time from 24 mo of age to age at first positive serum ELISA, and (4) time from 24 mo of age to age at culling for clinical JD. Cattle age at first test positive was recorded in months and at the time when fecal and serum samples were collected or when cattle were culled with clinical JD. Analysis was stratified by birth cohorts, and differences among strata were tested using the Wilcoxon and log-rank tests. The analysis was performed using the Lifetest procedure of SAS (SAS Institute Inc.).

The association between the recommended management practices and the incidence of JD was evaluated using a Cox regression model. Four models were built, with a separate model for each of the 4 primary outcomes of the study: (1) time from 24 mo of age to age at first positive from bacterial culture of feces, (2) time from 24 mo of age to age at first heavy shedding result from bacterial culture of feces, (3) time from 24 mo of age to age at first positive from serum ELISA, and (4) time from 24 mo of age to age at culling for clinical JD. Each of these outcomes was modeled using the following predictors: herd, source of the cattle (home raised or purchased), birth cohort, CRS, and 2-way interactions. A main effect model (with no interactions) was fitted for each outcome to obtain Martingale and Schoenfeld residuals and the baseline hazard functions for the birth cohort -2 . Martingale residuals were used to confirm the functional form of CRS as a continuous variable, and Schoenfeld residuals for each main covariate were obtained to evaluate the proportional hazard assumption. Schoenfeld residuals and baseline hazards were plotted against time using Gplot procedure of SAS (SAS Institute Inc.). Visual examination of the Schoenfeld residuals showed a trend to decrease with time for the variable CRS in all the models. Similarly, a visual examination of the baseline cumulative hazard functions suggested a decrease of the hazards with time. Finally, the interactions CRS and time showed statistical significance $(P<0.05)$ in all the models. We concluded that all models violated the proportional hazards assumption; therefore, all models included a time-dependent interaction of CRS and time. Because events occurring at the same time were very uncommon, ties were handled using the Breslow approximation. Birth cohort was included as a categorical variable instead of a continuous variable to allow comparison across birth cohorts and to avoid collinearity of this variable with CRS. A backward elimination model reduction was considered for nonsignificant variables $(P>0.05)$. Finally, a few outliers were found using likelihood displacement; however, they were not found to be influential on the model parameters when deleted. Models were fitted using the PHreg procedure of SAS (SAS Institute Inc.). After model reduction, all models included the same variables:

$$
h_{i j k}(t)=h_{0}(t) \times \exp \left[B_{1} x_{i}+B_{2} x_{j}+B_{3} x_{k}+B_{4}\left(x_{k} \times t\right)\right],
$$

where $h_{i j k}(t)=$ hazard ratio of the $i j k$ th case at time $t$; $h_{0}(t)=$ baseline hazard at time $t ; B_{1} x_{i}=$ hazard ratio for the $i$ th herd, where $i=1$ to 8 herds; $B_{2} x_{j}=$ hazard ratio for the $j$ th birth cohort, where $j=-2$ to 5 birth cohort; $B_{3} x_{k}=$ hazard ratio for the $k$ th total CRS, where $k=$ 0 to 200; and $B_{4}\left(x_{k} \times t\right)=$ time-dependent interaction indicating rate of changes of the hazard ratio for the $k$ th total CRS by time.

Age at Onset of $\boldsymbol{J D}$. The association of the control program with the age at onset of JD was evaluated using 4 different linear mixed models, where the ages of cattle in months at the first MAP bacterial culture positive result, first serum ELISA positive result, first MAP heavy fecal shedding result, and detection of clinical JD were used as dependent variables. This analysis used all cows that tested positive or had clinical JD during the study period. All models included birth cohorts as the explanatory variable and herd as the random effect variable. Because the purpose of this analysis was to evaluate the overall trend of the changes in age at first test positivity or clinical JD over successive birth cohorts, birth cohorts were treated as continuous variables instead of categorical variables, assuming linear association between birth cohorts and the outcomes. Model assumptions were evaluated using residual plots, and models were fitted using the Mixed procedure of SAS (SAS Institute Inc.).

\section{RESULTS}

Herds were enrolled in this study between 2000 and 2004, and they remained in the study between 5 and 


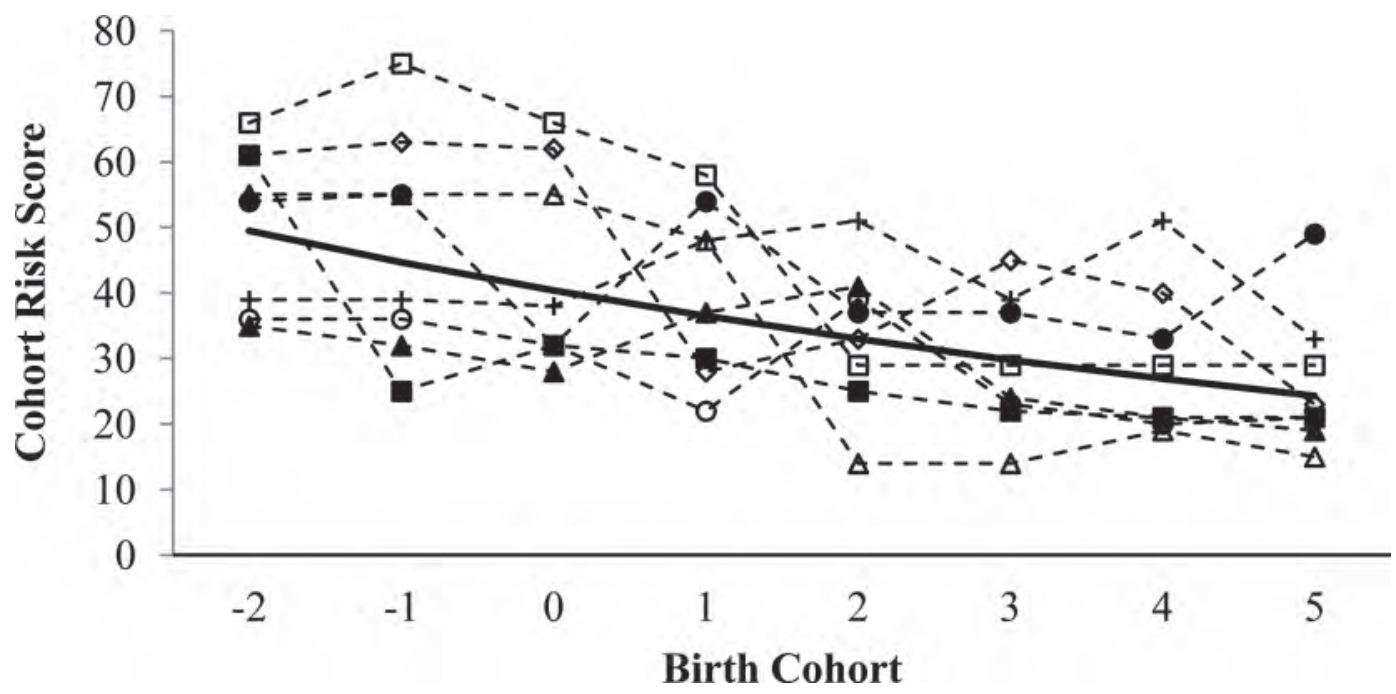

Figure 1. Cohort risk score (CRS) of Johne's disease by birth cohort in 8 dairy herds under Johne's disease control program in Minnesota. Dashed lines with different symbols indicate CRS for different herds and the solid line indicates the exponential regression across herds

$10 \mathrm{yr}$ (Table 1). The herd size of the farms enrolled in the study varied from 42 to 341 cows. The majority of study cows were Holstein breed with an average ME305 milk production that varied by herd from approximately 9,700 to $15,600 \mathrm{~kg}$ per lactation in the year of enrollment. The annual apparent prevalence of MAP bacterial culture positive and serum ELISA positive cows varied from 3.6 to $21.8 \%$ and from 3.2 to $28.5 \%$, respectively, in the year of enrollment, depending on the herd (Table 1).

The study population included 6,080 cows; however, 511 cows $(8.4 \%)$ were not included in any analysis because no test was performed on those cows, samples were mislabeled, or bacterial culture was contaminated with fungal overgrowth. During the study period, 12,787 bacterial cultures and 12,735 serum ELISA were performed. At the end of the study period, $7.26 \%$ of the 4,818 culled cows were culled due to clinical JD.

\section{Evaluation of Program Compliance}

The mean (SD) total ARAS was 48.5 (14.7) points at the beginning of the study and 30.6 (11.0) points at the end of the study. The mean (SD) risk assessment scores for the different production sections at the beginning and end of the study were 22.2 (8.7) and 15.9 (8.4) for calving area, 4.5 (6.1) and 2.0 (1.4) for preweaning heifers, 4.5 (4.6) and 3.5 (3.3) for postweaned heifers, 12.7 (3.6) and 6.6 (3.1) for bred heifers, and 2.8 (1.6) and 2.6 (2.0) for cows and bulls.

The mean (SD) CRS of the first and last birth cohort under study were 50.9 (12.4) and 26.5 (10.8) points.
Although we observed variations in the CRS by birth cohort and across herds, overall, all herds showed a numerical decrease in CRS. The results of the exponential regression showed a decrease of $9.7 \%$ (95\% CI: 5.3 to 13.8) in consecutive birth cohorts $(P<0.01$, Figure $1)$. The median (interquartile range) time between the date of fecal sample collection and the date of removal from the herd (culling date) was 8.4 mo (9.8) for bacterial culture-positive cows. The same values for light fecal shedders and heavy fecal shedders were $10.3 \mathrm{mo}$ (11.0) and $4.7 \mathrm{mo}(4.5)$.

\section{Association of Incidence and Recommended Management Practices}

The Kaplan-Meier curves and the time-dependent Cox regression analysis included 3,760 and 3,758 cows followed using MAP bacterial culture and JD serum ELISA, respectively, in the 8 birth cohorts and 8 herds. In total, 3,911 cows were observed for clinical signs of JD and included in these analyses. During the followup period, 432, 350,101, and 182 cows were positive for MAP bacterial culture, JD serum ELISA, MAP heavy fecal shedders, and clinical JD, respectively (Table 2).

The Wilcoxon and the log-rank tests of equality of Kaplan-Meier survival curves indicated differences in the incidence of MAP test positive and clinical JD across birth cohorts for time to first MAP bacterial culture positive results $(P<0.01$ and $P<0.05$, respectively), time to first JD serum ELISA positive result ( $P$ $<0.01$ and $P<0.01$, respectively), and time to clinical JD $(P<0.01$ and $P<0.05$, respectively $)$. The null 
Table 2. Number of cows followed and percentage of cattle censored by birth cohort and diagnostic test in 8 dairy herds under Johne's disease control program in Minnesota

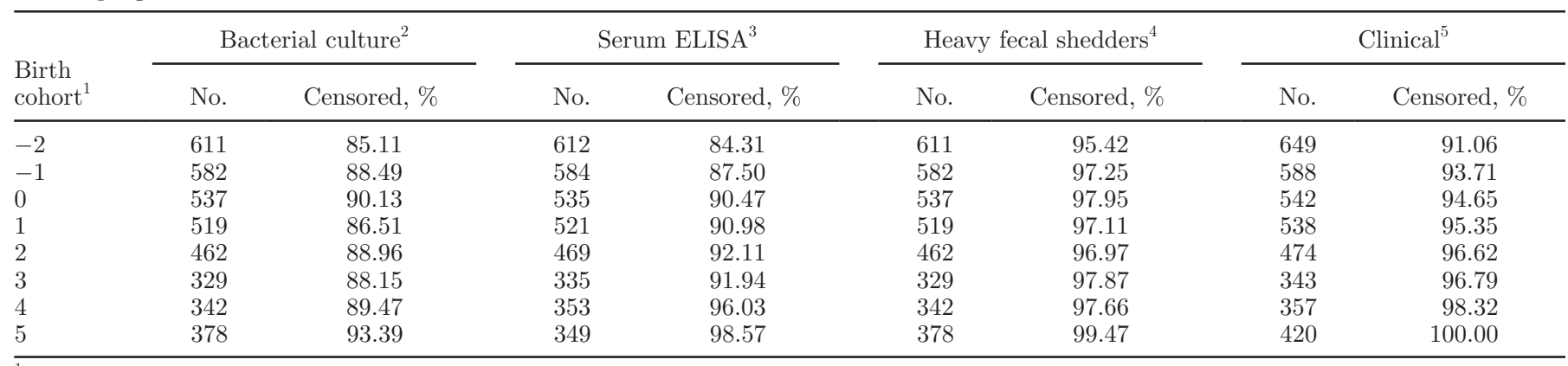

${ }^{1}$ Birth cohorts represent annual birth cohorts before and after the implementation of the disease control program (before: -2 and -1 ; after: 0 , $1,2,3,4$, and 5$)$.

${ }^{2}$ Mycobacterium avium ssp. paratuberculosis bacterial culture in Herrold's egg yolk medium.

${ }^{3}$ Johne's disease serum ELISA (Idexx Laboratories Inc., Westbrook, ME).

${ }^{4}$ Mycobacterium avium ssp. paratuberculosis bacterial culture in Herrold's egg yolk medium with diagnostic test results $>50 \mathrm{cfu} / \mathrm{slant}$.

${ }^{5}$ Clinical Johne's disease as recorded by herd managers.

hypothesis of no differences across birth cohort survival curves was not rejected for the time to first MAP heavy fecal shedding result (Figure 2).

In general, the percentage of survivals increased and the incidence of test positivity or clinical JD decreased in successive birth cohorts. The proportions (95\% CI) of cows that remained negative to bacterial culture at 36 and 48 mo of age, respectively, were 0.93 (0.91 to 0.95 ) and 0.86 (0.83 to 0.90 ) in birth cohort $-2,0.94$ (0.92 to 0.96 ) and 0.86 (0.83 to 0.90$)$ in birth cohort 1 , and 0.95 (0.93 to 0.98 ) and 0.85 (0.77 to 0.92$)$ in birth cohort 5 . Similar results were observed for serum ELISA positivity, MAP heavy fecal shedders, and clinical JD (Figure 2).

The variable "home raised or purchased cattle" and possible 2-way interactions were not significant in any of the survival models and therefore not included in the final models. The final time-dependent Cox regressions for the 4 outcomes (time to first MAP bacterial culture positive result, time to first JD serum ELISA positive result, time to first MAP heavy fecal shedding result, and time to clinical JD) included the same explanatory variables: herd, cohort, CRS, and the interaction between CRS and time.

Time to first MAP bacterial culture positive result was associated with birth cohort $(P<0.01)$. The instantaneous hazard of testing bacterial culture positive for birth cohorts $-1,0$, and 1 were similar to that of the reference birth cohort $(-2)$; however, the instantaneous hazard of testing bacterial culture positive decreased approximately $40 \%$ in birth cohort 2, and approximately $60 \%$ in birth cohorts 3,4 , and 5 compared with the reference birth cohort (Table 3). An increase in CRS was also associated with an increase in the instantaneous hazard of testing MAP bacterial cul- ture positive $(P<0.01)$. The instantaneous hazard of testing MAP culture positive increased 1.21-fold (95\% CI: 1.19 to 1.23) for every 1-point increase in the CRS; however, the strength of this association decreased over time $(P<0.01)$ by approximately 0.009 -fold $(95 \%$ CI: 0.008 to 0.01 ) per month of age.

Changes in the instantaneous hazard of MAP heavy shedding in feces were also associated with birth cohort $(P<0.05)$, CRS $(P<0.01)$, and the interaction between CRS and time $(P<0.01)$. No statistical differences in hazard ratios were found in birth cohorts -2 and $-1,0,1$, and 2 ; however, the hazard ratios of birth cohorts 3,4 , and 5 were lower than the same reference level (birth cohort -2; Table 3). The estimated increase in the instantaneous hazard of testing MAP heavy fecal shedding was 1.27 -fold (1.22 to 1.33 ) per unit of increase in CRS $(P<0.01)$; however, the magnitude of this association decreased 0.01 -fold $(0.008$ to 0.01$)$ by month of age $(P<0.01)$.

A similar time-dependent Cox regression to evaluate changes in the instantaneous hazard of testing MAP serum ELISA positive explained by birth cohorts also showed a relative constant decrease of the instantaneous hazard from birth cohort 0 compared with previous birth cohorts $(P<0.01$, Table 3$)$. The CRS was also associated $(P<0.01)$ with the instantaneous hazard of ELISA positive and this association was modified by time in the program $(P<0.01)$. The instantaneous hazard of testing serum ELISA positive increased 1.22fold (95\% CI: 1.20 to 1.25$)$ for each point of increase in the CRS; however, the strength of this association decreased 0.01-fold (0.009 to 0.01) per month of age.

Finally, the instantaneous hazard of clinical JD was associated with birth cohort $(P<0.01)$, CRS $(P<$ $0.01)$, and the interaction between CRS and time $(P$ 

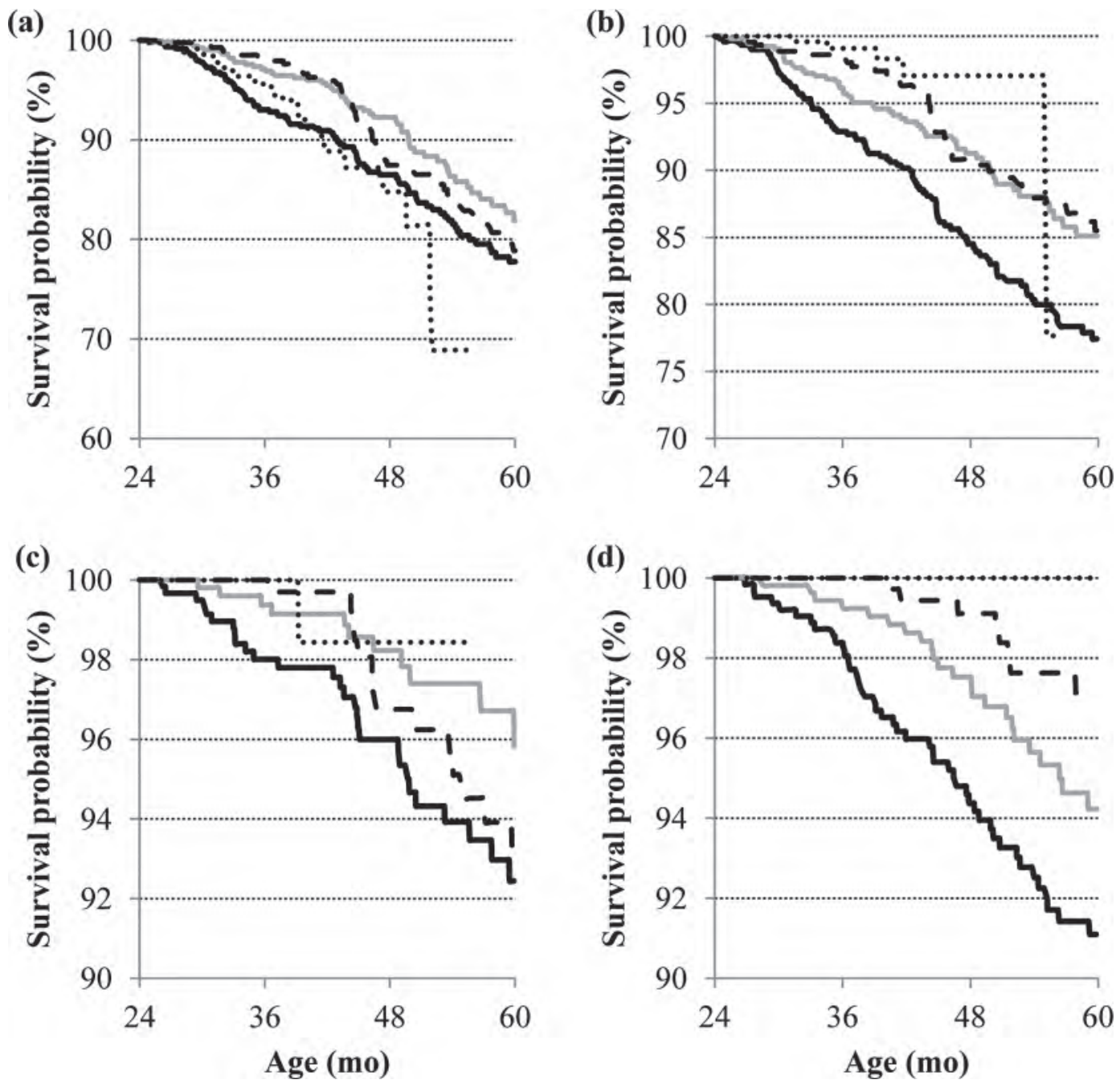

Figure 2. Kaplan-Meier survival curves for the time to (a) first Mycobacterium avium ssp. paratuberculosis positive bacterial culture, (b) first positive Johne's disease serum ELISA, (c) first Mycobacterium avium ssp. paratuberculosis heavy fecal shedder, and (d) clinical Johne's disease by annual birth cohorts in 8 dairy herds under Johne's disease control program in Minnesota. Annual birth cohorts represent birth cohort 2 yr before (solid black line), the year of implementation (solid gray line), and 2 (dotted line) and 5 (dashed line) yr after the implementation of a Johne's disease control program.

$<0.01)$. A reduction in the instantaneous hazard of clinical JD was observed from birth cohort 1 and later birth cohorts compared with the reference birth cohort (Table 3). The instantaneous hazard of clinical JD increased 1.33-fold (1.28 to 1.40) per unit of increase on the CRS; however, the magnitude of this association decreased 0.013 -fold (0.011 to 0.015$)$ per month of age.

\section{Age at Onset of JD}

The overall mean age (SD) of first MAP bacterial culture positive result was 46.8 (15.2) mo across all herds and cohorts. The mean age of first serum ELISA positive result, MAP heavy fecal shedding, and clinical JD were 45.7 (14.8), 49.5 (16.0), and 50.7 (14.1) mo, respectively. No overall tendency on the age at the first MAP bacterial culture positive, serum ELISA positive result, MAP heavy fecal shedding, and clinical JD was observed for successive birth cohorts.

\section{DISCUSSION}

Several management practices have been recommended to reduce transmission of JD in dairy cattle herds. Although a few studies have shown the effectiveness of some of these management practices on reducing the prevalence of JD (Jubb and Galvin, 2004; Collins et al., 2010; Nielsen and Toft, 2011), this study is the first to evaluate herd management practice changes in JD incidence in multiple herds, adjusting for 
Table 3. Hazard ratios (HR) and 95\% CI of test-positive Mycobacterium avium ssp. paratuberculosis (MAP) bacterial culture of feces, Johne's disease serum ELISA and bacterial culture heavy fecal shedder, and clinical Johne's disease, across birth cohorts in 8 dairy farms under a disease control program in Minnesota

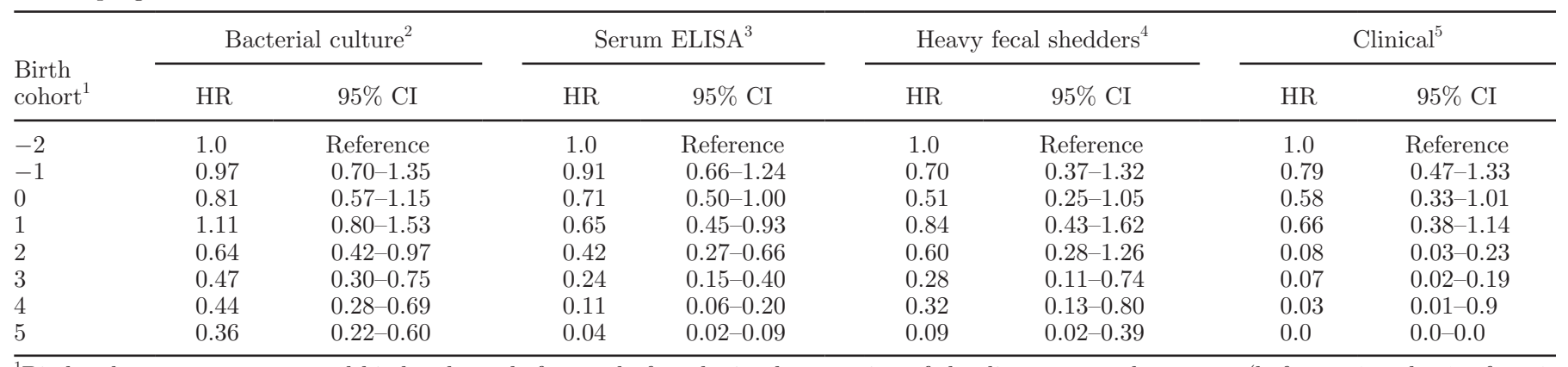

${ }^{1}$ Birth cohorts represent annual birth cohorts before and after the implementation of the disease control program (before: -2 and -1 ; after: 0 , $1,2,3,4$, and 5). Birth cohort -2 represents the reference level.

${ }^{2}$ Mycobacterium avium ssp. paratuberculosis bacterial culture in Herrold's egg yolk medium.

${ }^{3}$ Johne's disease serum ELISA (Idexx Laboratories Inc., Westbrook, ME).

${ }^{4}$ Mycobacterium avium ssp. paratuberculosis bacterial culture in Herrold's egg yolk medium with diagnostic test results $>50 \mathrm{cfu} / \mathrm{slant}$.

${ }^{5}$ Clinical Johne's disease as recorded by herd managers.

recommended management practice compliance. The Minnesota JDDHP was in operation for up to $10 \mathrm{yr}$, starting with herds enrolled in 2000, which was longer than other herds in the JDDHP. A partial evaluation of this program with 6 herds and 6 yr of follow-up was performed previously (Ferrouillet et al., 2009); however, the project included data from 2 additional herds and continued monitoring until 2009. Now that data collection for this project has concluded, a complete evaluation involving the 8 herds enrolled for the whole study period was needed.

This study was a prospective longitudinal field study, in which changes in herd management practices were recommended to reduce the transmission of JD. Compliance on the implementation of the recommended management practices was evaluated annually using the ARAS. Based on the ARAS, the level of disease exposure of each birth cohort was estimated and quantified using the CRS, as explained above. We observed an overall decrease in the CRS throughout years in the study and birth cohorts. When the first and the last cohort under study were compared, the CRS was decreased by almost $50 \%$. The CRS represented a subjective evaluation of the disease exposure of each birth cohort, and a reduction in the CRS by birth cohort indicated a decrease in disease exposure in successive birth cohorts.

Even though herd managers and owners were willing to participate in the control program and were enthusiastic about the program, none of them were able to implement all the recommended management practices to reduce the ARAS or CRS to zero. In fact, compliance with the recommended practices, evaluated using the ARAS, was similar to that observed in other Minnesota dairy herds enrolled in the VBJDCP, when the same period was compared (Wells et al., 2008). Analyses of the factors that affect the level of compliance of the recommended management practices were beyond the scope of this study; however, we expected a positive association between the level of compliance and the ease and feasibility of implementation of recommended practices in the herds and between the level of compliance and the within-herd prevalence of JD. All herds enrolled in this study showed numerical reductions in ARAS during the study period, and the observed decrease of the CRS across birth cohorts was a consequence of the reduction in ARAS. Similarly, a study in Michigan that used a similar risk assessment survey found that 6 out 7 dairy herds were able to reduce their risk score after 5 yr in a control program (Pillars et al., 2011). Another study of 54 herds in Australia that focused on calf rearing-related management practices to control JD found that only $18.5 \%$ of the herds followed all the main recommendations on calf management practices recommended to control JD (Ridge et al., 2005).

We hypothesize that a reduction in the CRS would decrease MAP exposure and effective transmission of JD to susceptible cattle and, consequently, a decrease in the JD incidence on successive birth cohorts should be expected. The study design, however, did not include cows not subject to disease control measures or herds that did not implement any changes in management practices to reduce disease transmission during the same study period. Because MAP exposure levels were quantified using the CRS for each birth cohort, within each herd, previous birth cohorts served as controls for the following birth cohorts, because they were exposed to different levels of MAP. 
The selected study herds were willing to participate in the JD control program and they perceived JD as an important disease in dairy production. To illustrate the perception of JD by Minnesota dairy producers, by 2006 , Minnesota was the US state with the highest proportion of dairy herds $(30.8 \%)$ enrolled in the VBJDCP at a national level (Wells et al., 2008). Even though herd managers showed interest of reducing the incidence of JD, the reduction was only observed 1 or 2 yr after the program formally started.

The unadjusted and nonparametric survival analysis using Kaplan-Meier curves indicated differences in the survival curves of bacterial culture and serum ELISA test positive or clinical JD across birth cohorts. In general, those birth cohorts that were born after the beginning of the control program showed a lower risk of testing bacterial culture or serum ELISA positive or being a clinical JD.

The hazard ratios of testing bacterial culture positive showed an important reduction (approximately $60 \%$ ) between birth cohorts 3 and 5 compared with previous birth cohorts. The main effect of changes in management practices on the reduction of the disease transmission was not observed in cows that were born within the first $2 \mathrm{yr}$ of the program (birth cohorts 0 and 1 ). During this period, most of the practical and economically feasible management changes recommended were likely implemented. The hazard ratios of testing bacterial culture positive for birth cohorts 3,4 , and 5 were almost constant, which may suggest that disease transmission reached an equilibrium point with the host and environment of the farms and (or) the presence of cows that passively fecal shed MAP to a level of detection by bacterial culture.

A reduction of the instantaneous hazard of testing bacterial culture positive was not observed until birth cohort 2 (cows that were born within the third year after the beginning of the program). In contrast to what was observed by the hazard ratios for bacterial culture positive cows along birth cohorts, the hazard ratios of serum ELISA positive cows showed an overall reduction from birth cohorts 1 to 5 . The reduction in the hazard ratios of serum ELISA positive along birth cohort was more constant (almost linear) in those cows born after the formal control program started (birth cohort 0). Although hazard ratios of bacterial culture positive and serum ELISA positive cows showed a decrease along birth cohorts, the decrease in the instantaneous hazard of serum ELISA positive cows occurred early compared with the decrease in bacterial culture positive hazard ratios. One explanation is that cows exposed to MAPcontaminated environments and passively fecal shedding MAP may lead to positive bacterial culture of feces (false positive), but without a humoral immune reac- tion detected by serum ELISA. Unfortunately, it was not possible to directly compare these ratios between tests, because they are on a relative scale with respect to an unknown reference baseline, and the baseline for each test may be different.

Hazard ratios of MAP fecal heavy shedders showed a reduction in cows that were born within the fourth year of the control program (birth cohort 2), and later, compared with the reference birth cohort $(-2)$. Study herd managers were asked to cull those cows that shed large amounts of MAP into the environment (MAP heavy fecal shedders) as soon as they were detected. The observed reduction in new cases of MAP heavy fecal shedders could be expected for several reasons: (1) decrease in JD transmission, (2) culling test positive cows before they became MAP heavy fecal shedders, or (3) premature culling of these cows because of decreased lactation performance.

A reduction in JD transmission in the herd as a consequence of the disease control program could have a direct effect on reducing the incidence of MAP fecal shedders and on reducing the proportion of MAP fecal shedders that later became MAP heavy fecal shedders. The reduction in the incidence of MAP heavy fecal shedders may also be explained as an indirect consequence of early detection and culling bacterial culture and serum ELISA positive cows before these cows became MAP heavy fecal shedders. Finally, MAP heavy fecal shedding is associated with reduced milk production of approximately 1,500 kg per lactation (Raizman et al., 2007); therefore, MAP heavy fecal shedders could have been culled because of low milk production before they were diagnosed as heavy fecal shedders.

Herd managers were aware of the importance of culling cows with clinical JD because of the negative effect of the disease on production performance (Raizman et al., 2007) and because these cows are likely shedding large amounts of MAP into the environment and transmitting the disease to susceptible cattle. Hazard ratios of clinical JD showed a constant decrease in instantaneous hazard after the third year of the program and, in fact, the hazard ratio of clinical JD in birth cohort 5 was zero, with no observed cases for that birth cohort.

All Cox regression models in this study had a significant time-dependent interaction with CRS, indicating that the association of CRS with the instantaneous hazard was not constant throughout the life of the cows. Cohort risk score was positively associated with the 4 different outcomes, indicating that an increase in the CRS was associated with an increasing instantaneous hazard of testing bacterial culture positive, serum ELISA positive, heavy shedding, or clinical JD. The estimated values of the time-dependent interaction indicated that the magnitude of the association 
between CRS and the 4 outcomes decreased with the age of the cows.

A decrease in the strength of the association between the level of implementation of the recommended management practices and the outcomes of the study during the life of the cows may be explained by the fact that susceptibility to JD is age dependent (Windsor and Whittington, 2010). After birth, calves are considered most susceptible and this susceptibility decreases when cattle become older. The risk assessment score of the herds accounted for this by placing $40 \%$ of the total possible score ( 80 out of 200 ) in the calving area. However, although adult cattle are less susceptible to JD, transmission of the disease among this age group may still occur, and, in fact, may play an important role in maintaining disease in herds with low frequency of the disease and in which disease transmission in calves has been minimized (Mitchell et al., 2008). Other factors that may contribute to maintaining JD in the herd may be the role that uncontrolled or unknown risk factors play in the dynamics of the disease. The importance of recommended management practices in controlling disease transmission may vary depending on the frequency of the disease. The management practices were recommended to minimize the risk of disease transmission based on the best current knowledge; however, at low disease frequency, uncontrolled and unknown risk factors may become proportionally more important, and may explain some levels of disease transmission in these herds after 5 to $10 \mathrm{yr}$ in the control program.

In this study, not all birth cohorts were followed for the same period. Older birth cohorts were followed for a longer period, which increased the probability of finding new test positive cows in older birth cohorts compared with younger birth cohorts. To control for the bias generated by differential follow-up time among birth cohorts, the analysis of the data considered that all cows were censored at 60 mo of age (approximately 3 lactations) or at the end of the study.

We also considered that the control program may not reduce transmission and incidence of the disease along birth cohorts, but instead may only delay the age of positive testing or culling for clinical JD. Censoring all cows at 60 mo of age controlled the differential followup time bias for the birth cohorts; however, this approach did not allow us to evaluate whether the control program reduced or delayed the disease transmission. The objective of the analysis of age at first positive tests or culling for clinical JD was to evaluate the existence of differential age of testing positive and culled for JD among birth cohorts.

All birth cohorts showed similar mean age at first positive test or clinical JD. The mean age at first fecal culture positive was similar to the mean age at first serum ELISA test positive; however, as expected, the mean age of MAP heavy fecal shedding and clinical JD was approximately 4 to 5 mo older. Even though, based on these results, it is not possible to make inferences about the progression of the disease at the individual cow level and study how test results change along with JD progression over time, we can make inferences at a population level about the expected age when cows become test positive or clinically ill if infected. In a herd where JD is prevalent and under a control program, the likelihood that a cow tests positive for MAP or becomes clinical JD during the first 3 lactations is higher than that of a cow in the same herd in the fourth or fifth lactation.

The annual testing scheme of the cows in this study resulted in an overestimation of the time to test positive, because positive cows could have tested positive earlier if they had been tested more frequently. Similarly, age at clinical JD may be overestimated because cows with clinical JD were recorded on the day that they were culled, and cows could have shown signs of clinical JD before the culling event. Even though the age at positive test or clinical JD was likely overestimated, this bias would not represent a differential misclassification, because the hazard ratio is a relative measure that compares the instantaneous hazard of testing positive of each birth cohort with an unknown baseline hazard of the reference birth cohort $(-2)$. Therefore, if the lag time between becoming positive or clinical JD and test detection as positive or culling is similar in all the birth cohorts, the estimated hazard ratios should be valid.

\section{CONCLUSIONS}

Although this observational study did not allow us to define causal associations between the implementation of the recommended management practices and the reduction of incidence of test-positive cattle and clinical JD, we can conclude that herds enrolled in the study were able to effectively reduce the incidence of test-positivity and clinical JD in successive birth cohorts and that this reduction was associated with a higher level of implementation of the recommended management practices suggested by the VBJDCP. The longitudinal nature of the study not only demonstrated a temporal association between changes in management practices and the incidence of test-positive cattle and clinical JD, but also estimated the strength of these associations. The results of this study reinforce the hypothesis that implementation of the recommended management practices reduces JD transmission, leading to a reduction in the incidence of disease. 


\section{ACKNOWLEDGMENTS}

We thank the study herd owners, managers, and employees for their participation and enthusiastic collaboration with the disease control program. We also thank the Minnesota Board of Animal Health district veterinarians and staff for their collaboration and support of the JD demonstration herds, and the Minnesota Veterinary Diagnostic Laboratory (St. Paul) for diagnostic testing and sample storage. We acknowledge the anonymous reviewers for their time and suggestions to improve this manuscript. Finally, we thank the USDA-APHIS Veterinary Services and the Johne's Disease Integrated Program funded by USDA National Institute for Food and Agriculture (Washington, DC) for providing partial funding for this study.

\section{REFERENCES}

Benedictus, A., R. M. Mitchell, M. Linde-Widmann, R. Sweeney, T. Fyock, Y. H. Schukken, and R. H. Whitlock. 2008. Transmission parameters of Mycobacterium avium subspecies paratuberculosis infections in a dairy herd going through a control program. Prev. Vet. Med. 83:215-227. http://dx.doi.org/10.1016/j.prevetmed.2007.07.008.

Collins, M. T., V. Eggleston, and E. J. B. Manning. 2010. Successful control of Johne's disease in nine dairy herds: Results of a six-year field trial. J. Dairy Sci. 93:1638-1643. http://dx.doi.org/10.3168/ jds.2009-2664.

Ferrouillet, C., S. J. Wells, W. L. Hartmann, S. M. Godden, and J. Carrier. 2009. Decrease of Johne's disease prevalence and incidence in six Minnesota, USA, dairy cattle herds on a long-term management program. Prev. Vet. Med. 88:128-137. http://dx.doi. org/10.1016/j.prevetmed.2008.08.001.

Gardner, I. A., S. S. Nielsen, R. J. Whittington, M. T. Collins, D. Bakker, B. Harris, S. Sreevatsan, J. E. Lombard, R. Sweeney, D. R. Smith, J. Gavalchin, and S. Eda. 2011. Consensus-based reporting standards for diagnostic test accuracy studies for paratuberculosis in ruminants. Prev. Vet. Med. 101:18-34. http://dx.doi. org/10.1016/j.prevetmed.2011.04.002.

Groenendaal, H., and D. T. Galligan. 2003. Economic consequences of control programs for paratuberculosis in midsize dairy farms in the United States. J. Am. Vet. Med. Assoc. 223:1757-1763. http:// dx.doi.org/10.2460/javma.2003.223.1757.

Hutchinson, L. J. 1996. Economic impact of paratuberculosis. Vet. Clin. North Am. Food Anim. Pract. 12:373-381.

Jubb, T. F., and J. W. Galvin. 2004. Effect of a test and control program for bovine Johne's disease in Victorian dairy herds 1992-2002. Aust. Vet. J. 82:228-232. http://dx.doi. org/10.1111/j.1751-0813.2004.tb12685.x.

Kudahl, A. B., S. S. Nielsen, and S. Østergaard. 2008. Economy, efficacy, and feasibility of a risk-based control program against paratuberculosis. J. Dairy Sci. 91:4599-4609. http://dx.doi. org/10.3168/jds.2008-1257.

Mitchell, R. M., R. H. Whitlock, S. M. Stehman, A. Benedictus, P. P. Chapagain, Y. T. Grohn, and Y. H. Schukken. 2008. Simulation modeling to evaluate the persistence of Mycobacterium avium ssp. paratuberculosis (MAP) on commercial dairy farms in the United States. Prev. Vet. Med. 83:360-380. http://dx.doi.org/10.1016/j. prevetmed.2007.09.006.

MNBAH. 2005. Minnesota Johne's Disease (JD) Control Program. Risk Assessment. Version 1, Nov 2005. Minnesota Board of Animal Health. Accessed May 17, 2011. http://www.bah.state.mn.us/ diseases/johnes/files/risk-assesment.pdf.

Nielsen, S. S., and N. Toft. 2011. Effect of management practices on paratuberculosis prevalence in Danish dairy herds. J. Dairy Sci 94:1849-1857. http://dx.doi.org/10.3168/jds.2010-3817.

Pillars, R. B., D. L. Grooms, J. C. Gardiner, and J. B. Kaneene. 2011. Association between risk-assessment scores and individual-cow Johne's disease-test status over time on seven Michigan, USA dairy herds. Prev. Vet. Med. 98:10-18. http://dx.doi.org/10.1016/j.prevetmed.2010.10.001.

Raizman, E. A., J. Fetrow, S. J. Wells, S. M. Godden, M. J. Oakes, and G. Vazquez. 2007. The association between Mycobacterium avium ssp. paratuberculosis fecal shedding or clinical Johne's disease and lactation performance on two Minnesota, USA dairy farms. Prev. Vet. Med. 78:179-195. http://dx.doi.org/10.1016/j. prevetmed.2006.10.006.

Ridge, S. E., I. M. Baker, and M. Hannah. 2005. Effect of compliance with recommended calf-rearing practices on control of bovine Johne's disease. Aust. Vet. J. 83:85-90. http://dx.doi. org/10.1111/j.1751-0813.2005.tb12204.x.

USDA-APHIS. 2008. Johne's disease on U.S. dairies, 1991-2007. USDA-APHIS, Fort Collins, CO

USDA-APHIS. 2010. Uniform Program Standards for the Voluntary Bovine Johne's Disease Control Program. Effective September 1, 2010. APHIS 91-45-016. Accessed Mar. 22, 2012. http://www. aphis.usda.gov/animal_health/animal_diseases/johnes/downloads/johnes-ups.pdf.

Wells, S. J., W. L. Hartmann, and P. L. Anderson. 2008. Evaluation of progress made by dairy and beef herds enrolled in the Minnesota Johne's Disease Control Program. J. Am. Vet. Med. Assoc. 233:1920-1926. http://dx.doi.org/10.2460/javma.233.12.1920.

Wells, S. J., R. H. Whitlock, and C. J. Lindeman. 2002. Evaluation of bacteriologic culture of pooled fecal samples for detection of Mycobacterium paratuberculosis. Am. J. Vet. Res. 63:1207-1211. http://dx.doi.org/10.2460/ajvr.2002.63.1207.

Windsor, P. A., and R. J. Whittington. 2010. Evidence for age susceptibility of cattle to Johne's disease. Vet. J. 184:37-44. http:// dx.doi.org/10.1016/j.tvjl.2009.01.007. 